Pacific Journal of Mathematics

SIMPLE PERIODIC MODULES OF TWISTED CHEVALLEY 


\title{
SIMPLE PERIODIC MODULES OF TWISTED CHEVALLEY GROUPS
}

\section{Peter Fleischmann and Jens Carsten Jantzen}

\begin{abstract}
Consider a finite twisted Chevalley group constructed over a field of prime characteristic and its representations over an algebraically closed field of the same characteristic. In this paper we classify all those irreducible representations that are periodic, i.e., that have a periodic projective resolution. There is always the Steinberg module that is both simple and projective. We show that there are further periodic simple modules only for groups of types ${ }^{2} A_{2}$ and ${ }^{2} B_{2}$.
\end{abstract}

0. Introduction. Let $K$ be an algebraically closed field of characteristic $p>0$ and $\Gamma$ a finite group with $p|| \Gamma \mid$. A finite dimensional $K \Gamma$-module $M$ is called periodic, if it has a periodic minimal projective resolution. Of course projective modules are also periodic, but the non-projective ones are characterized by their complexity $c_{\Gamma}(M)$ being one. Here we understand complexity in the sense of Alperin [1].

In this article we look at the case where $\Gamma$ is an "almost simple" twisted group of Lie type, defined over a finite field of the same characteristic $p$. We will give a classification of all periodic simple modules for these groups and the finite simple groups related to them.

It is a well-known fact that all finite groups of Lie type have a unique simple projective module, the Steinberg module St. We will prove in this paper that, if $\Gamma$ is not of type ${ }^{2} A_{2}$ or ${ }^{2} B_{2}$, then the only periodic simple $K \Gamma$-module is St. With the classification for ${ }^{2} A_{2}$ in [6], [7], and for ${ }^{2} B_{2}$ in part 3 of this paper, we will achieve a full classification of simple periodic modules for twisted groups.

The corresponding classification for non-twisted Chevalley groups was recently accomplished in [9], [10]. Together with the initial classification for the type $A_{1}$ in [15], the final result is that for finite groups of Lie type there are simple periodic modules, other than St, only for groups of type $A_{1},{ }^{2} A_{2}$ and ${ }^{2} B_{2}$.

To state our results more precisely, we have to introduce some notations which we take over from [5]. For conceptual reasons we prefer working with universal groups:

So let $K$ be an algebraic closure of the prime field $\mathbb{F}_{p}$, let $G$ be an almost simple, simply connected and connected affine algebraic 
group over $K$, defined and split over $\mathbb{F}_{p}$. Let $F$ be a Frobenius endomorphism of $G$, as in [5], $T$ an $F$-stable maximal torus of $G$ with character group $X(T)$ and $R \subset X(T)$ be the (indecomposable) root system of $G$. We choose a basis $\Pi=\left\{\alpha_{1}, \alpha_{2}, \ldots, \alpha_{l}\right\}$ of $R$ and assume $F$ to induce a non-trivial automorphism $\tau$ of the Dynkin diagram of $G$. So either $\tau$ is of order 3 and $R$ of type $D_{4}$ or $\tau$ is of order 2 and $R$ of type $A_{l}(l>1), D_{l}(l>3), E_{6}$, or $B_{2}, G_{2}, F_{4}$ (in which cases $p$ must be $2,3,2$ respectively).

Attached to $F$ are natural numbers $i, e \in \mathbb{N}$ such that $F^{i}(t)=t^{p^{e}}$ for all $t \in T$ and the number $q:=p^{e / i}$ is uniquely determined by $G$ and $F$. The group $G^{F}$ of $F$-fixed points is either a finite (universal) Steinberg group (usually denoted by ${ }^{3} D_{4}\left(q^{3}\right)_{\text {s.c. }},{ }^{2} A_{l}\left(q^{2}\right)_{\text {s.c. }}$, ${ }^{2} D_{l}\left(q^{2}\right)_{\text {s.c. }}$ or ${ }^{2} E_{6}\left(q^{2}\right)_{\text {s.c. }}$ ) or a Ree or Suzuki group (usually denoted by ${ }^{2} B_{2}\left(q^{2}\right),{ }^{2} F_{4}\left(q^{2}\right),{ }^{2} G_{2}\left(q^{2}\right)$ where $q^{2}=2^{2 m+1}$ resp. $q^{2}=3^{2 m+1}$ for some $m$ ).

Excluding the cases (a) ${ }^{2} A_{2}(4)_{\text {s.c. }}$, (b) ${ }^{2} B_{2}(2)_{\text {s.c. }}$, (c) ${ }^{2} G_{2}(3)_{\text {s.c. }}$ and (d) ${ }^{2} F_{4}(2)_{\text {s.c. }}$, their quotient by the center is simple (see [19], Theorem 34 , pg. 188) and is usually denoted by ${ }^{3} D_{4}\left(q^{3}\right),{ }^{2} A_{l}\left(q^{2}\right)$ etc. (or by ${ }^{3} D_{4}(q),{ }^{2} A_{l}(q)$, according to the taste of the author.)

The groups in (a) and (b) are solvable, whereas in (c) and (d) their commutator subgroups have index 3 and 2 and are simple non-abelian finite groups $\left(\cong \mathrm{PSl}_{2}(8)\right.$ in (c), $\cong$ Tits' simple group in (d)) ([19], pg. 188).

In this framework the main theorem of this article reads as follows:

0.1. TheOREM. Let $\Gamma$ be $G^{F}, G^{F} / Z\left(G^{F}\right)$ or $\left(G^{F}\right)^{\prime}$, then all periodic simple $K \Gamma$-modules are projective if and only if $R$ is not of type $A_{2}$ or $B_{2}$, in which case they are isomorphic to $\mathrm{St}_{G^{F}}$ or to a Clifford component of it, if $\left(G^{F}\right)^{\prime}<G^{F}$.

In $\S 1$ we will reduce the problem for the Steinberg groups to the case $G^{F} \cong{ }^{2} A_{3}\left(q^{2}\right)_{\text {s.c. }} \cong \mathrm{SU}_{4}\left(q^{2}\right)$, which will be dealt with in $\S 2$. Factoring out the center, which does not affect the Sylow- $p$-structure, leads to the result for the simple groups. Section 3 is devoted to the Ree and Suzuki groups.

1. The reduction to $\mathrm{SU}_{4}$. In this section we exclude the cases $B_{2}, G_{2}$ and $F_{4}$ but keep the previous notations. So all roots in $R$ have the same length. We assume moreover $T$ to be defined and split over $\mathbb{F}_{p}$. So the usual Frobenius endomorphism $x \mapsto x^{p}$ on $K$ gives rise to an endomorphism $F_{0}$ of $G$, acting on $T$ via $F_{0}(t)=t^{p}$ for 
all $t \in T$. There is an automorphism of $G$ that has the same order as $\tau$, commutes with $F$, stabilizes $T$ and induces on $\Pi \subseteq X(T)$ the same map as $\tau$. If this automorphism is also denoted by $\tau$, then we can write $F=\tau \circ F_{0}^{n}=F_{0}^{n} \circ \tau$ for some integer $n>0$; in this case $q=p^{n}$. We denote the fundamental dominant weights of $R$ by $\lambda_{\alpha}$ and set $\rho:=\sum \lambda_{\alpha}$ where the sum is over all simple roots. Let $X(T)^{+}$ be the set of dominant weights and set for all $n>0$ :

$$
X_{n}(T):=\left\{\lambda=\sum r_{\alpha} \lambda_{\alpha} \in X(T) \mid 0 \leq r_{\alpha}<p^{n} \text { for all } \alpha \in \Pi\right\} .
$$

For any $\lambda \in X(T)^{+}$let $V(\lambda)$ be the Weyl module for $G$ with highest weight $\lambda$ and $L(\lambda)$ its unique simple quotient. One has $V\left(\left(p^{n}-1\right) \rho\right)=L\left(\left(p^{n}-1\right) \rho\right)$ for all $n$; this module is often called the $n$th Steinberg module and denoted by $\mathrm{St}_{n}$.

For any $G$-module $M$ and any $r \in \mathbb{N}$ let $M^{(r)}$ denote the $G$-module got from $M$ by twisting the $G$-action with $F_{0}^{r}$. If $\mu=\sum p^{i} \mu_{i}$ where $i$ ranges from 0 to $n-1$ and $\mu_{i} \in X_{1}(T)$, then

$$
L(\mu) \cong L\left(\mu_{0}\right) \otimes L\left(\mu_{1}\right)^{(1)} \otimes \cdots \otimes L\left(\mu_{n-1}\right)^{(n-1)}
$$

by Steinberg's tensor product theorem [18].

According to another theorem of Steinberg [18] the $L(\lambda)$ with $\lambda \in$ $X_{n}(T)$ remain irreducible when regarded as a representation of $G^{F}$, and each simple $K G^{F}$-module is isomorphic to exactly one $L(\lambda)$ with $\lambda \in X_{n}(T)$.

The $n$th Steinberg module $\mathrm{St}_{n}=L\left(\left(p^{n}-1\right) \rho\right)$ is known to be projective as a $K G^{F}$-module. So in the case of the Steinberg groups our main theorem amounts to:

(1.3) Theorem. Suppose that $G$ is a Steinberg group not of type $A_{2}$. Then $L(\lambda)$ is not a periodic $K G^{F}$-module for any $\lambda \in X_{n}(T), \lambda \neq$ $\left(p^{n}-1\right) \rho$.

Using the corresponding results for non-twisted groups of type $A_{l}$, $l>1$ (proved in [9]) we reduce the proof of this theorem to the case of ${ }^{2} A_{3}$ (i.e. to the groups $\mathrm{SU}_{4}\left(q^{2}\right)$ ) that will be dealt with in $\S 2$.

(1.4) Proposition. If Theorem 1.3 holds in the case ${ }^{2} A_{3}$, then it holds in all cases.

Proof. Suppose that $L(\lambda)$ is periodic. Then for any subgroup $H$ of $G^{F}$ any direct summand $M$ of $L(\lambda)$ as a $K H$-module is periodic. So we prove the proposition by constructing in each case some $H$ and $M$ such that $M$ is not periodic for $K H$. 
Here and in the following we will abbreviate a weight $\lambda=\sum r_{i} \lambda_{\alpha_{t}}$ by $\left(r_{1}, r_{2}, \ldots, r_{l}\right)$, where $\Pi=\left\{\alpha_{1}, \ldots, \alpha_{l}\right\}$.

Case 1. Type $A_{l}, l>3$. Here the Dynkin diagram is:

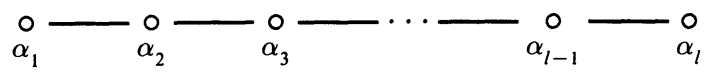

We consider $G^{F} \cong \mathrm{SU}_{l+1}\left(q^{2}\right)$ with respect to the hermitian form given by the unit matrix.

Then $c_{1}: g \mapsto\left(\begin{array}{ll}g & 0 \\ 0 & 1\end{array}\right)$ and $c_{2}: g \mapsto\left(\begin{array}{ll}1 & 0 \\ 0 & g\end{array}\right)$ define two canonical embeddings of $\mathrm{SU}_{l}\left(q^{2}\right) \rightarrow \mathrm{SU}_{l+1}\left(q^{2}\right)$. If $L_{J}$ denotes the Levi subgroup for $J \subseteq \Pi$ in $G$, then the image $\operatorname{Im} c_{1}$ is contained in the derived group $L_{\Pi \backslash\left\{\alpha_{1}\right\}}^{\prime}:=L_{1}$ and $\operatorname{Im} c_{2} \subseteq L_{\Pi \backslash\left\{\alpha_{1}\right\}}^{\prime}:=L_{2}$; hence we can apply a theorem of Smith [17]:

If $\lambda=\left(r_{1}, \ldots, r_{l}\right) \in X_{n}(T)$ then $\left.L(\lambda)\right|_{L_{l}}=L\left(\lambda_{i}\right) \oplus M_{i}$ where $L\left(\lambda_{i}\right)$ is an irreducible module for $L_{i}$ with highest weight $\lambda_{i}$ and $M_{i}$ is some other $L_{i}$ module. We get $\lambda_{1}=\left(r_{1}, r_{2}, \ldots, r_{l-1}\right)$ and $\lambda_{2}=\left(r_{2}, r_{3}, \ldots, r_{l}\right)$. See also [16] for a proof of these facts.

Furthermore $L_{i}$ is simply connected of type $A_{l-1}$. If $\lambda \neq$ $\left(p^{n}-1\right) \rho$, then we may assume without loss of generality that $\lambda_{1} \neq$ $\left(p^{n}-1\right)\left(\rho-\lambda_{\alpha_{l}}\right)$. Then $L\left(\lambda_{1}\right)$ is a simple periodic and non-projective $\mathrm{SU}_{l}\left(q^{2}\right)$ module in contradiction to the induction hypothesis.

Case 2. Type ${ }^{2} D_{l}\left(q^{2}\right)_{\text {s.c. }}$

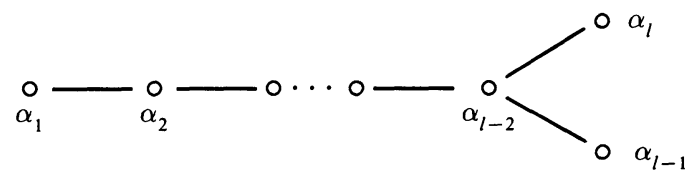

Let $L_{i}$ be the derived group of the Levi subgroup corresponding to $J_{i}$, where $J_{i}=\left\{\alpha_{i}, \alpha_{i+1}\right\}$ for $i<l-2$ and $J_{l-2}=\left\{\alpha_{l-2}, \alpha_{l-1}, \alpha_{l}\right\}$. Each $L_{i}$ is $F$-stable and simply connected; one has $L_{i}^{F} \cong A_{2}(q)_{\text {s.c. }}$. for $i<l-2$ and $\left(L_{l-2}\right)^{F} \cong{ }^{2} A_{3}\left(q^{2}\right)_{\text {s.c. }}$. If again $\lambda=\left(r_{1}, r_{2}, \ldots, r_{l}\right)$, then by Smith's theorem $\left.L(\lambda)\right|_{L_{i}^{F}}=L\left(\lambda_{i}\right) \oplus M_{i}$ as above with $\lambda_{i}=$ $\left(r_{i}, r_{i+1}\right)$ for $1 \leq i \leq l-3$, and $\lambda_{l-2}=\left(r_{l-2}, r_{l-1}, r_{l}\right)$. Again, since $L(\lambda) \neq \mathrm{St}_{n}$, one of these $L\left(\lambda_{i}\right)$ 's must be non-projective and hence non-periodic for $L_{i}^{F}$, by the corresponding result for $A_{2}(q)$ in [9] and the hypothesis.

Case 3. Type ${ }^{2} E_{6}\left(q^{2}\right)_{\text {s.c. }}$ :

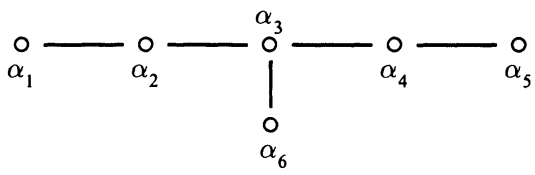


The same kind of argument applies here with $J_{1}=\Pi \backslash\left\{\alpha_{6}\right\}, J_{2}=$ $\left\{\alpha_{3}, \alpha_{6}\right\}$ and $\left(L_{J_{1}}\right)^{F} \cong{ }^{2} A_{5}\left(q^{2}\right),\left(L_{J_{2}}\right)^{F} \cong A_{2}(q)$, using Case 1 and the result in [9].

Case 4. Type ${ }^{3} D_{4}\left(q^{3}\right)_{\text {s.c. }}$ :

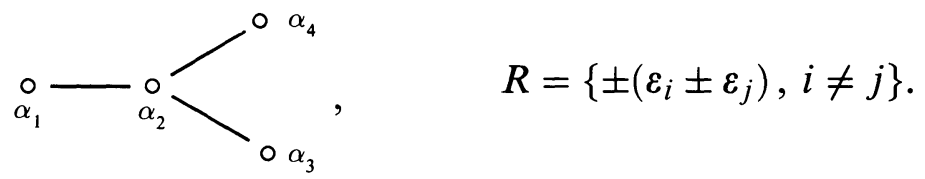

The Weyl group $W(G, T)$ acts on $\mathbb{Z}^{4}$ as permutations of the $\pm \varepsilon_{i}$ 's with an even number of sign changes.

If $\alpha_{1}:=\varepsilon_{1}-\varepsilon_{2}, \alpha_{2}:=\varepsilon_{2}-\varepsilon_{3}, \alpha_{3}:=\varepsilon_{3}-\varepsilon_{4}, \alpha_{4}:=\varepsilon_{3}+\varepsilon_{4}$, then the $\tau$-fixed points $\alpha_{2}, \alpha_{1}+\alpha_{2}+\alpha_{3}+\alpha_{4}=\varepsilon_{1}+\varepsilon_{3}=: \beta, \alpha_{1}+2 \alpha_{2}+\alpha_{3}+\alpha_{4}=$ $\varepsilon_{1}+\varepsilon_{2}$, form an $A_{2}$-subsystem of $R$. Moreover $W$ maps

$$
\begin{array}{ll}
\left(\alpha_{2}, \beta\right) \mapsto\left(\alpha_{2}, \alpha_{1}\right) & \text { under } \quad\left(\begin{array}{cccc}
1 & 2 & 3 & 4 \\
1 & -3 & -2 & 4
\end{array}\right) \\
\left(\alpha_{1}, \alpha_{2}\right) \mapsto\left(\alpha_{2}, \alpha_{3}\right) & \text { under }\left(\begin{array}{cccc}
1 & 2 & 3 & 4 \\
2 & 3 & 4 & 1
\end{array}\right) \\
\left(\alpha_{1}, \alpha_{2}\right) \mapsto\left(\alpha_{2}, \alpha_{4}\right) & \text { under }\left(\begin{array}{cccc}
1 & 2 & 3 & 4 \\
2 & 3 & -4 & -1
\end{array}\right)
\end{array}
$$

If $U_{\alpha}$ denotes the root subgroup of $G$, then $U_{\alpha_{2}}, U_{\beta}$ are $F$-stable and $U_{\alpha_{2}}^{F}, U_{\beta}^{F} \cong \mathbb{F}_{q}^{+}$. Let $H$ be the subgroup $\left\langle U_{ \pm \alpha_{2}}, U_{ \pm \beta}\right\rangle \leq G$; then $H$ is of type $A_{2}$ and $H^{F}$ of type $A_{2}(q)$. For $i=1,3,4$ let $J_{i}=$ $\left\{\alpha_{i}, \alpha_{2}\right\}$; hence $L_{J_{i}}^{\prime}=: L_{i}$ is of type $A_{2}$ but not $F$-stable. Let now $L(\lambda) \neq \mathrm{St}_{n}$, with $\lambda=\left(r_{1}, r_{2}, r_{3}, r_{4}\right)$.

Then $\left.L(\lambda)\right|_{L_{i}}=L\left(\lambda_{i}\right) \oplus M_{i}$, where $\lambda_{i}=\left(r_{2}, r_{1}\right)$ resp. $\left(r_{1}, r_{2}\right)$.

For each $i$ there is some $w_{i} \in W$ such that $w_{i}(H)=w_{i} H w_{i}^{-1}$ is contained in $L_{i}$. Since $\left.L(\lambda)\right|_{H^{F}}$ is periodic, also the restrictions $\left.L(\lambda)\right|_{w_{i}\left(H^{F}\right)}$ must be periodic. This can be seen for instance looking at the rank variety in the sense of [3], [4] and the fact that rank varieties of conjugate groups are isomorphic. Since $w_{i}(H)$ is contained in $L_{i}$, also $\left.L\left(\lambda_{i}\right)\right|_{w_{i}\left(H^{F}\right)}$ is periodic. But $w_{i}\left(H^{F}\right) \cong A_{2}(q)$ and $r_{i}<q$, so $L\left(\lambda_{i}\right)$ is also irreducible for $w_{i}\left(H^{F}\right)$, which forces $r_{i}$ and $r_{2}$ to be $q-1$ by the result in [9]. This gives a contradiction.

2. The Case $\mathrm{SU}_{4}$. Keep all assumptions and notations from $\S 1$ and assume that $R$ is of type $A_{3}$. 
(2.1) LemMA. Let $\mu=\sum_{l=0}^{n-1} p^{l} \mu_{l} \in X_{n}(T)$ with all $\mu_{l} \in X_{1}(T)$. If $L(\mu)$ is a periodic $K G^{F}$-module, then each $\mu_{l}$ has one of the following forms :

(a) $(p-1, p-1, p-1)=(p-1) \rho$,

(b) $(p-1, p-1, i)$ with $0 \leq i<p-1$,

(b') $(i, p-1, p-1)$ with $0 \leq i<p-1$,

(c) $(p-1, i, j)$ with $0 \leq i, j, i+j=p-2$,

(c) $(i, j, p-1)$ with $0 \leq i, j, i+j=p-2$,

(d) $(p-1, i, p-1)$ with $0 \leq i<p-1$,

(e) $(i, p-1, j)$ with $0 \leq i, j<p-1$,

(f) $(p-2-i, i, p-2-i)$ with $0 \leq i<p-1$.

Proof. Suppose $\mu=\left(m_{1}, m_{2}, m_{3}\right)$. The same argument as in the proof of (1.4), Case 1 shows that $L\left(\left(m_{1}, m_{2}\right)\right)$ and $L\left(\left(m_{2}, m_{3}\right)\right)$ are periodic as $\mathrm{KSU}_{3}\left(q^{2}\right)$-modules. The classification of these modules in [7] yields now the claim.

(2.2) Lemma. Let $\lambda \in X_{1}(T)$ be of type (d), (e), (f) in Lemma (2.1). Then $\operatorname{dim} L(\lambda)$ is not divisible by $p^{3}$.

Proof. The formulae for the characters of the $L(\lambda)$ in [12] $\S 7$ imply: $\operatorname{dim} L(p-1, i, p-1)=\operatorname{dim} V(p-1, i, p-1)$

$$
-\operatorname{dim} V(p-(i+2), i, p-(i+2))
$$

for $0 \leq i<p-1$;

$\operatorname{dim} L(i, p-1, j)=\operatorname{dim} V(i, p-1, j)$

$$
\begin{array}{r}
-\operatorname{dim} V(p-(j+2), p-1, p-(i+2)) \\
+\operatorname{dim} V(p-(i+2), i+j+1-p, p-(j+2)) \\
\quad \text { for } 0 \leq i, j<p-1, i+j>p-2 ;
\end{array}
$$

$\operatorname{dim} L(i, p-1, j)=\operatorname{dim} V(i, p-1, j)$ for $0 \leq i, j, i+j=p-2$; $\operatorname{dim} L(i, p-1, j)=\operatorname{dim} V(i, p-1, j)$

$$
\begin{array}{r}
-\operatorname{dim} V(j, p-(i+j+3), i) \\
\quad \text { for } 0 \leq i, j, i+j<p-2 ;
\end{array}
$$

$\operatorname{dim} L(p-2-i, i, p-2-i)=\operatorname{dim} V(p-2-i, i, p-2-i)$ 
By Weyl's dimension formula

$\operatorname{dim} V(r, s, t)=(r+1)(s+1)(t+1)(r+s+2)(s+t+2)(r+s+t+3) / 12$.

In each case one sees immediately that $p^{3}$ does not divide $\operatorname{dim} V(\mu)$ for any $\mu$. In the case $\lambda=(p-1, i, p-1)$ one gets

$$
\begin{aligned}
& \operatorname{dim} L(\lambda) \equiv 2 \operatorname{dim} V(\lambda) \not \equiv 0 \quad\left(\bmod p^{3}\right) \quad \text { for } p \geq 5, \\
& \operatorname{dim} L(\lambda) \equiv 2 \operatorname{dim} V(\lambda) \not \equiv 0 \quad\left(\bmod p^{2}\right) \quad \text { for } p=3, \\
& \operatorname{dim} L(\lambda)=14 \not \equiv 0 \quad(\bmod 8) \quad \text { for } p=2 .
\end{aligned}
$$

The other cases are similar and are left to the reader.

Recall the definition of the rank variety $V_{E} M$ of a $K E$-module $M$ for any elementary abelian $p$-group $E$ as in [3] or [4]. So it is a homogeneous subvariety of the vector space $K^{m}$ where $m$ is the rank of $E$. It is called linear, if it is a linear subspace of $K^{m}$.

(2.3) LEMMA. Let $E$ be an elementary abelian p-subgroup of $G^{F}=$ $\mathrm{SU}_{4}\left(q^{2}\right)$ and let $\mu \in X_{1}(T)$ be of type (b) or (c) in Lemma (2.1). Then

$$
V_{E} L(\mu)=V_{E} L((p-1,0, p-2))
$$

Proof. Set $C^{*}=\left\{\left(r_{1}, r_{2}, r_{3}\right) \in X(T) \mid-1 \leq r_{1}, r_{2}, r_{3}, r_{1}+r_{2}+r_{3} \leq\right.$ $p-3\}$. Then $C^{*}$ is a fundamental domain for the "dot" operation (i.e., $w \cdot \mu=w(\mu+\rho)-\rho$ ) of $W_{p}$ on $X$, where $W_{p}$ is the affine Weyl group (as in [14], II, 6.1). So there is a unique $\mu^{\prime} \in W_{p} \cdot \mu \cap C^{*}$ and a unique $\lambda^{\prime} \in W_{p} \cdot \lambda \cap C^{*}$ where $\lambda=(p-1,0, p-2)$; in fact $\lambda^{\prime}=(-1,-1,0)$.

We want to use the translation functors $T_{\lambda^{\prime}}^{\mu^{\prime}}$ and $T_{\mu^{\prime}}^{\lambda^{\prime}}$, cf. [14], II, 7.6. More precisely, we shall show that $T_{\lambda^{\prime}}^{\mu^{\prime}} L(\lambda) \cong L(\mu)$ and $T_{\mu^{\prime}}^{\lambda^{\prime}} L(\mu) \cong L(\lambda)$. As $T_{\lambda^{\prime}}^{\mu^{\prime}} L(\lambda)$ is a direct summand of $L(\lambda) \otimes M$ for a suitable finite dimensional $G$-module $M$, and $T_{\mu^{\prime}}^{\lambda^{\prime}} L(\mu)$ a direct summand of $L(\mu) \otimes M^{*}$, elementary properties of the rank variety (cf. [3]) yield $V_{E} L(\lambda)=V_{E} L(\mu)$.

If $\mu$ is of type (c) in Lemma (2.1), then $\lambda$ and $\mu$ belong to the same facet for $W_{p}$. In this case $T_{\lambda^{\prime}}^{\mu^{\prime}} L(\lambda) \cong L(\mu)$ and $T_{\mu^{\prime}}^{\lambda^{\prime}} L(\mu) \cong L(\lambda)$ are special cases of [14], II, 7.15.

Suppose now that $\mu$ is of type b) in Lemma (2.1), i.e. $\mu=(p-1$, $p-1, i)$ for some $i, 0 \leq i<p-1$. Then $\mu^{\prime}=(-1, i, p-(i+2))$. One has $V(\lambda)=L(\lambda)$ and $V(\mu)=L(\mu)$ by [11], p. 120 or by [12]. 
We can compute the formal characters of $T_{\lambda^{\prime}}^{\mu^{\prime}} L(\lambda)$ and $T_{\mu^{\prime}}^{\lambda^{\prime}} L(\mu)$ using [14], II, 7.8. Let $s_{i}=s_{\alpha_{i}}$ be the reflection with respect to $\alpha_{i}$ (for $i=1,2,3)$ and let $s \in W_{p}$ be the affine reflection with $s(r, s, t)=$ $(r+s+t-p, p-t, p-s)$ for all $(r, s, t) \in X(T)$. Then the stabilizer of $\mu^{\prime}$ (under the dot action) in $W_{p}$ consists of $\left\{1, s_{1}, s, s_{1} s, s s_{1}\right.$, $\left.s_{1} s s_{1}\right\}$, that of $\lambda^{\prime}$ of $\left\{1, s_{1}, s_{2}, s_{1} s_{2}, s_{2} s_{1}, s_{1} s_{2} s_{1}\right\}$. The intersection of these groups is $\left\{1, s_{1}\right\}$, a system of representatives modulo this intersection is $\left\{1, s_{2}, s_{1} s_{2}\right\}$ in $\operatorname{Stab}\left(\lambda^{\prime}\right)$ and $\left\{1, s, s_{1} s\right\}$ in $\operatorname{Stab}\left(\mu^{\prime}\right)$.

The element $w \in W_{p}$ with $w(r, s, t)=(p-r, r+s+t, p-t)$ has the property that $\mu=w \cdot \mu^{\prime}$ and $\lambda=w \cdot \lambda^{\prime}$. One has

$$
\begin{aligned}
w s_{2} \cdot \mu^{\prime} & =(p-(i+2), p-1,-1), \\
w s_{1} s_{2} \cdot \mu^{\prime} & =(p+i, p-(i+2),-1) .
\end{aligned}
$$

These weights are not dominant, but become dominant after adding $\rho=(1,1,1)$. Therefore they contribute 0 to the sum in [14], II, 7.12, and we get $T_{\lambda^{\prime}}^{\mu^{\prime}} L(\lambda)=T_{\lambda^{\prime}}^{\mu^{\prime}} V(\lambda) \cong V(\mu) \cong L(\mu)$. Furthermore

$$
\begin{aligned}
w s \cdot \lambda^{\prime} & =(2 p-2, p-1,-1), \\
w s_{1} s \cdot \lambda^{\prime} & =(0,2 p-2,-1) .
\end{aligned}
$$

The same argument as above implies

$$
T_{\mu^{\prime}}^{\lambda^{\prime}} L(\mu)=T_{\mu^{\prime}}^{\lambda^{\prime}} V(\mu) \cong V(\lambda)=L(\lambda) .
$$

For $\alpha \in R$, let $U_{\alpha}=\left\{x_{\alpha}(t) \mid t \in K\right\}$ be the root subgroup of $G$ with root homomorphism $x_{\alpha}: K \mapsto G$. We set:

$$
H:=U_{\alpha_{1}} \circ U_{\alpha_{1}+\alpha_{2}} \circ U_{\alpha_{2}+\alpha_{3}} \circ U_{\alpha_{1}+\alpha_{2}+\alpha_{3}} \cong\left(K^{+}\right)^{4} \text {. }
$$

If $\left\{u_{1}, u_{2}, \ldots, u_{n}\right\},\left\{v_{1}, v_{2}, \ldots, v_{2 n}\right\}$ denote bases of $\mathbb{F}_{q}$ and $\mathbb{F}_{q^{2}}$ over $\mathbb{F}_{p}$ respectively, then let $x_{1}\left(u_{i}\right):=x_{\alpha_{2}}\left(u_{i}\right)$,

$$
x_{2}\left(v_{j}\right):=x_{\alpha_{1}+\alpha_{2}}\left(v_{j}\right) x_{\alpha_{2}+\alpha_{3}}\left(v_{j}^{q}\right), \quad x_{3}\left(u_{k}\right):=x_{\alpha_{1}+\alpha_{2}+\alpha_{3}}\left(u_{k}\right) .
$$

The group

$$
E:=H^{F}=\left\langle x_{1}\left(u_{i}\right) x_{2}\left(v_{j}\right) x_{3}\left(u_{k}\right) \mid 1 \leq i, k \leq n ; 1 \leq j \leq 2 n\right\rangle
$$

is then an elementary abelian $p$-subgroup of rank $4 n$ of $G^{F}=$ $\mathrm{SU}_{4}\left(q^{2}\right)$. 
(2.4) LEMMA. Let $\lambda \in X_{1}(T)$ be one of the weights in Lemma (2.1). Then

$$
\begin{gathered}
\operatorname{dim} V_{E} L(\lambda) \geq 4 n-2 \text { for } \lambda \neq(p-1) \rho \\
\operatorname{dim} V_{E} L((p-1) \rho) \geq 4 n-4
\end{gathered}
$$

Proof. Let $d:=\operatorname{dim} V_{E} L(\lambda)$; then by [3], Proposition 5.1, there exists a "shifted subgroup" $E^{\wedge} \leq K E^{*}$, which is elementary abelian of order $p^{4 n-d}$ such that $\left.L(\lambda)\right|_{E^{\wedge}}$ is free. In particular $p^{4 n-d}$ divides $\operatorname{dim} L(\lambda)$, which proves the claim for the weights of type (d), (e) and (f) by (2.2).

By (2.3) we are left with the weights $(p-1,0, p-2)$ and $(p-1) \rho$; in both cases $L(\lambda)=V(\lambda)$. Now let $J:=\left\{\alpha_{1}, \alpha_{2}\right\} \subseteq \Pi$; then $H$ can also be described as the unipotent radical $U_{J}$ of the standard parabolic subgroup $P_{J}=U_{J} \rtimes L_{J} \leq G$. If $\lambda=(r, s, t)$, then by Smith's theorem $\left.L(\lambda)\right|_{L_{J}}=L(\lambda)^{U_{J}} \oplus X$, where $L(\lambda)^{U_{J}}$ is the space of $U_{J}$-fixed points, which is also an irreducible module for $L_{J}^{\prime} \cong$ $A_{1}(K)_{\text {s.c. }} \times A_{1}(K)_{\text {s.c. }}$, with highest weight $(r, t)$, hence has dimension $(r+1)(t+1)$.

Since $E \subseteq U_{J}$ and $E^{\wedge}$ is generated by elements of the form $1+\sum a_{i}\left(x_{i}-1\right)$ with $a_{i} \in K, x_{i} \in E$, it is clear that $L(\lambda)^{U_{J}} \subseteq L(\lambda)^{E^{\wedge}}$, which has dimension $\operatorname{dim} L(\lambda) / p^{4 n-d}$ because $L(\lambda)$ is $E^{\wedge}$-free. So we get $p^{4 n-d} \leq \operatorname{dim} L(\lambda) /(r+1)(t+1)$. Now we apply Weyl's dimension formula and obtain $p^{4 n-d} \leq p^{2}(p+1) / 6$ for $\lambda=(p-1,0, p-2)$ and $p^{4 n-d} \leq p^{4}$ for $\lambda=(p-1) \rho$.

(2.5) Proposition. If $M$ is a periodic simple $\mathrm{KS} U_{4}\left(q^{2}\right)$-module, then

$$
M \cong \mathrm{St}_{n}
$$

Proof. By [3] 5.6, $V_{E}(X \otimes Y)=V_{E}(X) \cap V_{E}(Y)$ for $K E$-modules $X$ and $Y$. It is easy to see that $\operatorname{dim} V_{E}\left(L(\mu)^{(r)}\right)=\operatorname{dim} V_{E}(L(\mu))$, so if $M\left(\not \mathrm{St}_{n}\right)$ is written in the form of (1.2), then at least for one $\mu_{i}$ we must have $\operatorname{dim} V_{E}\left(L\left(\mu_{i}\right)^{(i)}\right) \geq 4 n-2$ by (2.4). Since all rank varieties $V_{E}$ are homogeneous affine subvarieties of $K^{4 n}$ the intersection formula for homogeneous varieties implies

$$
\begin{aligned}
\operatorname{dim} V_{E} M & =\operatorname{dim} \bigcap_{i=0}^{n-1} V_{E}\left(L\left(\mu_{i}\right)^{(i)}\right) \\
& \geq(n-1)(4 n-4)+4 n-2-(n-1) 4 n \geq 2,
\end{aligned}
$$

which contradicts periodicity by [3], 7.6 and 8.1 . 
REMARK. This proposition together with (1.4) implies Theorem (1.3), and it is clear, since $G^{F}=\left(G^{F}\right)^{\prime}$ if type (a) of the exceptions is excluded, how to get $(0.1)$.

3. The Ree and Suzuki groups. In this section we deal with the cases, where $R$ is of type $G_{2}, F_{4}$ and $B_{2}$, which were excluded earlier. Here twisted groups of Lie type can exist only if the characteristic of $K$ is 3,2 and 2 respectively, and $\tau$ always has order 2 , interchanging the long and short roots.

Proposition (3.1). Let $R$ be $G_{2}$ or $F_{4}$, char $K=3$ or 2 respectively and $\Gamma=G^{F}, G^{F} / Z\left(G^{F}\right)$, or $\left(G^{F}\right)^{\prime}$; then every periodic simple $K \Gamma$-module is projective, and either isomorphic to $\mathrm{St}_{G^{F}}$ or to a Clifford component of $\left.\mathrm{St}_{G^{F}}\right|_{\Gamma}$ if $\Gamma=\left(G^{F}\right)^{\prime} \triangleleft G^{F}$ and $\Gamma \neq G^{F}$.

Proof. We first prove this for $\Gamma=G^{F}$. By a result of Steinberg [18], $\S 12$, an analogue of (1.2) applies here, with $X_{1}(T)$ replaced by $X_{1}(T)^{\prime}=: X_{1}^{\prime}=\left\{\mu=\sum r_{\alpha} \lambda_{\alpha} \in X_{1}(T) \mid \alpha \in \Pi\right.$ and $r_{\alpha}=0$ if $\alpha$ is a long root $\}$.

Case 1. $G^{F}={ }^{2} G_{2}\left(q^{2}\right)_{\text {s.c. }}$ with $q^{2}=3^{n}, n=2 m+1$ and $\operatorname{char} K=$ $3, R=\{ \pm \alpha, \pm(2 \alpha+3 \beta), \pm(\alpha+3 \beta)$ (long roots), $\pm \beta, \pm(\alpha+\beta)$, $\pm(\alpha+2 \beta)$ (short roots) $\}$, and the Dynkin diagram is:

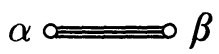

Here $X_{1}(T)^{\prime}=\{(0,0),(0,1),(0,2)\}$ and $\operatorname{dim} L(\lambda)=1,7,27$ for $\lambda \in X_{1}(T)^{\prime}$ respectively. See [18], $\S 12$.

Let $E=\left\{x(u, v) \mid u, v \in \mathbb{F}_{3^{n}}\right\}$ with

$$
x(u, v):=x_{\alpha+3 \beta}(u) x_{\alpha+\beta}\left(u^{3^{m}}\right) x_{2 \alpha+3 \beta}(v) x_{\alpha+2 \beta}\left(v^{3^{m}}\right) .
$$

Then $E$ is elementary abelian of rank $2 n$. With regard to the dimensions it is enough to look at $\lambda=(0,2)$. Then $L(\lambda)$ has the following "monomial basis" with highest weight vector $v_{\lambda}^{+}$:

$$
\left\{x_{-(\alpha+\beta)}^{n_{1}} X_{-(\alpha+2 \beta)}^{n_{2}} X_{-\beta}^{n_{3}} \circ v_{\lambda}^{+} \mid 0 \leq n_{i} \leq 2\right\},
$$

where $X_{-\gamma}$ are root vectors in $\operatorname{Lie}(G)$.

If $\gamma$ is any positive root, $x_{\gamma}: K \rightarrow G$ the corresponding root homomorphism and $V_{\mu}$ a weight subspace to the weight $\mu$ of some $G$ module $V$, then one knows:

$$
x_{\gamma}(t) \circ v_{\mu} \in \bigoplus_{i \in \mathbb{N}} V_{\mu+i \gamma} .
$$


Since $\lambda$ is the highest weight of $L(\lambda)$ and $E$ consists of unipotent elements, $E$ must fix the three basis vectors $v_{\lambda}^{+}, X_{-\beta} v_{\lambda}^{+}, X_{-\beta}^{2} v_{\lambda}^{+}$. If $d:=\operatorname{dim} V_{E}(L(0,2))$, then as in the proof of $(2.4)$, a suitable shifted elementary abelian $E^{\wedge} \leq(K E)^{*}$ with $\left|E^{\wedge}\right|=3^{2 n-d}$ acts freely on $L(0,2)$ and we get $\operatorname{dim} L(0,2) /\left|E^{-}\right|=3^{d-2 n+3} \geq 3$ and hence $d \geq$ $2 n-2$. By elementary properties of rank varieties, $V_{E}(L(\lambda)) \cong K^{2 n}$ for $\lambda=(0,0)$ or $(0,1)$. The analogue of $(1.2)$ together with the intersection theorem for affine varieties yields $\operatorname{dim} V_{E}(M) \geq 2$ for all $M \neq \mathrm{St}_{G^{F}}$.

Case 2. $G^{F}={ }^{2} F_{4}\left(q^{2}\right), q^{2}=2^{n}, n=2 m+1$ and $\operatorname{char} K=2$. Here $X_{1}(T)^{\prime}=\{(0,0,0,0),(0,0,0,1),(0,0,1,0),(0,0,1,1)\}$ with dimensions for $L(\lambda): 1,26,246,2^{12}$ respectively (see [20]). Let $\theta: K \rightarrow K, x \mapsto x^{2^{m}}$; we consider the following elements of $R^{+}\left(\Pi=\left\{\alpha_{1}, \alpha_{2}, \alpha_{3}, \alpha_{4}\right\}\right)$ :

$$
\begin{array}{ll}
\gamma_{1}:=\alpha_{1}+2 \alpha_{2}+3 \alpha_{3}+2 \alpha_{4} ; & \gamma_{2}:=2 \alpha_{1}+3 \alpha_{2}+4 \alpha_{3}+2 \alpha_{4} ; \\
\delta_{1}:=\alpha_{1}+2 \alpha_{2}+3 \alpha_{3}+\alpha_{4} ; & \delta_{2}:=\alpha_{1}+3 \alpha_{2}+4 \alpha_{3}+2 \alpha_{4} ; \\
\varepsilon_{1}:=\alpha_{1}+2 \alpha_{2}+2 \alpha_{3}+\alpha_{4} ; & \varepsilon_{2}:=\alpha_{1}+2 \alpha_{2}+4 \alpha_{3}+2 \alpha_{4} ; \\
\varphi_{1}:=\alpha_{1}+\alpha_{2}+2 \alpha_{3}+\alpha_{4} ; & \varphi_{2}:=\alpha_{1}+2 \alpha_{2}+2 \alpha_{3}+2 \alpha_{4} ; \\
\psi_{1}:=\alpha_{1}+\alpha_{2}+\alpha_{3}+\alpha_{4} ; & \psi_{2}:=\alpha_{1}+\alpha_{2}+2 \alpha_{3}+2 \alpha_{4} .
\end{array}
$$

Notice that $\gamma_{1}, \delta_{1}, \ldots$ are short and $\gamma_{2}, \delta_{2}, \ldots$ are long. Let

$$
\begin{array}{ll}
x_{1}\left(u_{1}\right):=x_{\gamma_{1}}\left(u_{1}\right) \circ x_{\gamma_{2}}\left(u_{1}^{2 \theta}\right) ; & x_{2}\left(u_{2}\right):=x_{\delta_{1}}\left(u_{2}\right) \circ x_{\delta_{2}}\left(u_{2}^{2 \theta}\right) ; \\
x_{3}\left(u_{3}\right):=x_{\varepsilon_{1}}\left(u_{3}\right) \circ x_{\varepsilon_{2}}\left(u_{3}^{\theta}\right) ; & x_{4}\left(u_{4}\right):=x_{\varphi_{1}}\left(u_{4}\right) \circ x_{\varphi_{2}}\left(u_{4}^{\theta}\right) ; \\
x_{5}\left(u_{5}\right):=x_{\psi_{1}}\left(u_{5}\right) \circ x_{\psi_{2}}\left(u_{5}^{2 \theta}\right) ; & \text { with } u_{i} \in \mathbb{F}_{2^{n}} .
\end{array}
$$

Notice that these elements commute because of char $K=2$, and hence the group $E:=\left\langle x_{i}\left(u_{i}\right) \mid i=1,2,3,4,5 ; u_{i} \in \mathbb{F}_{2^{n}}\right\rangle$ is an elementary abelian subgroup of $G^{F}$ of rank $5 n$.

For $L(0,0,1,1)$ there is a monomial basis, consisting of elements of the form: $X_{-\omega_{1}}^{n_{1}} \circ \cdots \circ X_{-\omega_{12}}^{n_{12}} v_{\lambda}^{+}$with $n_{i} \in\{0,1\}$, where $\left\{\omega_{1}, \omega_{2}, \ldots, \omega_{12}\right\}$ is the set of short positive roots.

The 7 short roots $\alpha_{3}, \alpha_{2}+\alpha_{3}, \alpha_{1}+\alpha_{2}+\alpha_{3}, \alpha_{4}, \alpha_{3}+\alpha_{4}, \alpha_{2}+\alpha_{3}+$ $\alpha_{4}, \alpha_{2}+2 \alpha_{3}+\alpha_{4}$ have not full support in $\Pi$. Since the generators of $E$ are products of $x_{\gamma}$ 's where $\gamma$ is a positive root with full support, an argument similar to that of Case 1 shows that basis vectors involving only monomials in those 7 roots have to be fixed by $E$ and hence by any shifted subgroup $E^{\wedge}$ of $K E^{*}$. 
Since there are $2^{7}$ such monomials, $\operatorname{dim} L(0,0,1,1)^{E^{\wedge}} \geq 2^{7}$. If $d=\operatorname{dim} V_{E}(L(0,0,1,1))$ then again we can choose $E^{\wedge}$ to act freely on $L(0,0,1,1)$ and to be of order $2^{5 n-d}$. So we get $\operatorname{dim} L(0,0,1,1) /\left|E^{-}\right|=2^{12-5+d} \geq 2^{7}$; hence $d \geq 5 n-5$. Since all other $V_{E}(L(\lambda))$ for $\lambda \in X_{1}(T)^{\prime}$ have dimension $\geq 5 n-1$ (4 does not divide $\operatorname{dim} L(\lambda)$ for $\lambda \neq(0,0,1,1))$, the analogue of $(1.2)$ yields:

$$
\operatorname{dim} V_{E}(M) \geq 4 \quad \text { for all } M \neq \mathrm{St}_{G^{F}}
$$

Proof for $G^{F} / Z\left(G^{F}\right)$ and $G^{F^{\prime}}$. As $Z\left(G^{F}\right)$ is a $p^{\prime}$-subgroup of $G^{F}$ the same results hold for $\Gamma=G^{F} / Z\left(G^{F}\right)$. Now let $G^{F^{\prime}} \neq G^{F}$; then $m=0, n=1$ and $q^{2}=\left|G^{F} / G^{F^{\prime}}\right|=p=3$ in Case 1 and $=2$ in Case 2.

By Clifford's theorem $\left.\mathrm{St}\right|_{G^{F^{\prime}}}$ is completely reducible. Let $V$ be a simple direct summand of $\mathrm{St}_{G^{F^{\prime}}}$, with inertia group $I:=I_{G^{F}}(V)=$ $\left\{g \in G^{F} \mid V^{g} \cong V\right.$ as $G^{F^{\prime}}$ modules $\}$. Then $I=G^{F^{\prime}}$. Otherwise by [8] $\S 9,(9.9), V=\left.W\right|_{G^{F^{\prime}}}$ for a simple $G^{F}$ module $W$, which is isomorphic to $\mathrm{St}_{G^{F}}$, since $V$ is projective; but then $W$ had to be a direct summand of the induced module $V^{G^{F}}$ since $W$ is projective, which contradicts the indecomposability of $V^{G^{F}}$ given by Green's theorem [8] §16. So $\left.\mathrm{St}\right|_{G^{F}} \cong \bigoplus_{g \in G^{F} / G^{F^{\prime}}} V^{g}$ and we claim that each periodic simple $G^{F^{\prime}}$ module is isomorphic to one of these $V^{g}$ 's.

Let $S$ be an irreducible periodic module for $G^{F^{\prime}}$. If $I=I_{G^{F}}(S)=$ $G^{F}$ then again $S=W_{G^{F^{\prime}}}$ for a simple $G^{F}$ module $W$. In Case $1, E \subseteq G^{F^{\prime}}$ and in Case $2 E \cap G^{F^{\prime}}$ has rank $\geq 4$; hence 3 or $2^{3}$ divides $\operatorname{dim} W$ respectively which forces $W$ to be $\cong \mathrm{St}_{G^{F}}$, leading to a contradiction. Hence $I=G^{F^{\prime}}$ which implies that $S^{G}$ is simple periodic for $G^{F}$. So $S^{G} \cong \mathrm{St}_{G^{F}}$ and $S \cong V^{g}$ for a $g \in G^{F}$ by Frobenius reciprocity. In particular $S$ is projective.

We conclude the treatment of twisted groups of Lie type, by computing the rank varieties of simple modules for $G^{F}={ }^{2} B_{2}\left(q^{2}\right)_{\text {s.c. }}$, which includes a classification of periodic simple modules, i.e. those whose variety has dimension 1 .

Let $q^{2}=2^{n}, n=2 m+1, \operatorname{char} K=2, \theta:=(?)^{2}, \Pi=\{\alpha, \beta\}$. The Dynkin diagram is $\Longrightarrow$. Let $P:=\left\{x(t, u) \mid t, u \in \mathbb{F}_{2^{2 m+1}}\right\} \in$ $\operatorname{Syl}_{2}\left(G^{F}\right)$, with $x(t, u)=x_{\alpha}(t) x_{\beta}\left(t^{\theta}\right) x_{\alpha+2 \beta}(u) x_{\alpha+\beta}\left(t^{1+\theta}+u^{\theta}\right)$; then $|P|=2^{4 m+2}$ and $Z(P)=\{x(0, u)\}$ is maximal elementary abelian; 
let $D:=Z(P)$. Notice that there is only one conjugacy class of maximal elementary abelian 2-subgroups in $G^{F}$. Here $X_{1}(T)^{\prime}=$ $\{(0,0),(0,1)\}$ with dimensions 1 and 4 respectively.

(3.2) Proposition. Let $G^{F}={ }^{2} B_{2}\left(q^{2}\right)_{\text {s.c. }}$ and $M$ be an irreducible $K G^{F}$-module; then $V_{E}(M)$ is linear and $\operatorname{dim} V_{E}(M)=n-s$, where $s$ is the number of factors $\lambda_{i}=(0,1)$ in the tensor product

$$
M=\bigotimes_{i=0}^{n-1} L\left(\lambda_{i}\right)^{(i)}, \quad \lambda_{i} \in X_{1}(T) .
$$

Proof. We need only to consider $L(0,1)$ which has a basis $B=$ $\left\{v^{+}, X_{-\beta} v^{+}, X_{-(\alpha+\beta)} v^{+}, X_{-\beta} X_{-(\alpha+\beta)} v^{+}\right\}$. A straightforward computation shows, that

$$
\begin{aligned}
& x(0, u) v^{+}=v^{+} ; \quad x(0, u) X_{-\beta} v^{+}=X_{-\beta} v^{+} \\
& x(0, u) X_{-(\alpha+\beta)} v^{+}=u^{\theta} v^{+}+X_{-(\alpha+\beta)} v^{+} \\
& x(0, u) X_{-\beta} X_{-(\alpha+\beta)} V^{+}=u v^{+}+u^{\theta} X_{-\beta} X_{-(\alpha+\beta)} V^{+} .
\end{aligned}
$$

Hence $x(0, u)$ is represented with respect to $B$ by

$$
\left[\begin{array}{cccc}
1 & & & \\
0 & 1 & 0 & \\
u^{\theta} & 0 & 1 & \\
u & u^{\theta} & 0 & 1
\end{array}\right]
$$

Let now $\left\{u_{i}\right\}$ be an $\mathbb{F}_{2}$-basis of $\mathbb{F}_{2^{n}}$, then $\left\{x\left(0, u_{i}\right)=: x_{i}\right\}$ is a basis of $E$. For $\mathbf{a}=\left(a_{1}, a_{2}, \ldots, a_{n}\right) \in K^{n}$ we define $u_{\mathbf{a}}:=1+\sum a_{i}\left(x_{i}-1\right)$. Then we get, using Lemma 4.2 of [3]:

$$
V_{E}(L(0,1))=\left\{\mathbf{a} \in K^{n} \mid \mathrm{rk} u_{\mathbf{a}}-1<\frac{1}{2} 4\right\}=\left\{\mathbf{a} \in K^{n} \mid \sum a_{i} u_{i}^{\theta}=0\right\} .
$$

The analogue of (1.2) now gives the result.

REMARK. It may be interesting to notice that non-projective simple periodic modules only occur in finite groups of Lie type, if the group has a split $B N$-pair of rank one. But ${ }^{2} G_{2}$ shows, they need not necessarily occur in this case.

\section{REFERENCES}

[1] J. L. Alperin, Periodicity in groups, Illinois J. Math., 21 (1977), 776-783.

[2] J. L. Alperin and L. Evens, Representations, resolutions and Quillen's dimension theorem, J. Pure Appl. Algebra, 22 (1981), 1-9. 
[3] J. F. Carlson, The varieties and the cohomology ring of a module, J. Algebra, 85 (1983), 104-143.

[4] , Module varieties and cohomology rings of finite groups, Vorlesungen aus dem FB Mathematik der Universitaet Essen, 13 (1985).

[5] R. W. Carter, Finite Groups of Lie Type: Conjugacy Classes and Complex Characters, New York etc. 1985 (Wiley).

[6] P. Fleischmann, Periodic simple modules for $\mathrm{SU}_{3}\left(q^{2}\right)$ in the describing characteristic $p \neq 2$, Math. Z., 198 (1988), 555-568.

[7] _- The complexities and rank varieties of the simple modules of ${ }^{2} A_{2}\left(q^{2}\right)$ in the natural characteristic, J. Algebra, 121 (1989), 399-408.

[8] B. Huppert and N. Blackburn, Finite Groups II, Berlin etc. 1982 (Springer).

[9] I. Janiszczak, Irreducible periodic modules over $\operatorname{SL}(m, q)$ in the describing characteristic, Comm. in algebra, 15 (1987), 1375-1391.

[10] I. Janiszczak and J. C. Jantzen, Simple periodic modules over Chevalley groups, to appear.

[11] J. C. Jantzen, Darstellungen halbeinfacher algebraischer Gruppen und zugeordnete kontravariante Formen, Bonner math. Schriften, 63 (1973).

[12] __ Zur Charakterformel gewisser Darstellungen halbeinfacher Gruppen und Lie-Algebren, Math. Z., 140 (1974), 127-149.

[13] _ Modular Representations of Reductive Groups, in Group Theory, Beijing 1984, Springer Lecture Notes, 1185 (1986), 118-154.

[14] _ Representations of Algebraic Groups, Orlando etc. 1987, (Academic Press).

[15] A. V. Jeyakumar, Periodic modules for the groups $\operatorname{SL}(2, q)$, Comm. in Algebra, 8 (1980), 1721-1735.

[16] G. M. Seitz, Representations and maximal subgroups of finite groups of Lie type, Geometriae Dedicata, 25 (1988), 391-406.

[17] S. D. Smith, Irreducible modules and parabolic subgroups, J. Algebra, 75 (1982), 286-289.

[18] R. Steinberg, Representations of algebraic groups, Nagoya Math. J., 22 (1963), 33-56.

[19] _ Lectures on Chevalley Groups, Yale Univ. (1968).

[20] F. D. Veldkamp, Representations of algebraic groups of type $F_{4}$ in characteristic 2 , J. Algebra, 16 (1970), 326-339.

Received July 25, 1988.

UNIVERSITAET-GHS-ESSEN

D-4300 EsSEN, W. GERMANY

AND

UNIVERSITAET

D-2000 Hamburg 13, W. Germany

Current address for J. C. Jantzen: University of Oregon Eugene, OR 97403 


\section{PACIFIC JOURNAL OF MATHEMATICS EDITORS}

\author{
V. S. VARADARAJAN \\ (Managing Editor) \\ University of California \\ Los Angeles, CA 90024-1555-05 \\ Herbert Clemens \\ University of Utah \\ Salt Lake City, UT 84112 \\ ThOMAs ENRIGHT \\ University of California, San Diego \\ La Jolla, CA 92093
}

R. FINN

Stanford University

Stanford, CA 94305

HeRmanN FlaschKa

University of Arizona

Tucson, AZ 85721

VAUGHAN F. R. JoNES

University of California

Berkeley, CA 94720

SteVen KerCKhofF

Stanford University

Stanford, CA 94305
ROBION KIRBY

University of California

Berkeley, CA 94720

C. C. Moore

University of California

Berkeley, CA 94720

HAROLD STARK

University of California, San Diego

La Jolla, CA 92093

\section{ASSOCIATE EDITORS}
R. ARENS
E. F. BECKENBACH
B. H. NeumanN
F. Wolf
K. YoshidA
(1906-1982)
(1904-1989)

\section{SUPPORTING INSTITUTIONS}

UNIVERSITY OF ARIZONA
UNIVERSITY OF BRITISH COLUMBIA
CALIFORNIA INSTITUTE OF TECHNOLOGY
UNIVERSITY OF CALIFORNIA
MONTANA STATE UNIVERSITY
UNIVERSITY OF NEVADA, RENO
NEW MEXICO STATE UNIVERSITY
OREGON STATE UNIVERSITY
UNIVERSITY OF ARIZONA
UNIVERSITY OF BRITISH COLUMBIA
UNIVERSITY OF CALIFORNIA
MONTANA STATE UNIVERSITY
NEW MEXICO STATE UNIVERSITY

\author{
UNIVERSITY OF OREGON \\ UNIVERSITY OF SOUTHERN CALIFORNIA \\ STANFORD UNIVERSITY \\ UNIVERSITY OF HAWAII \\ UNIVERSITY OF TOKYO \\ UNIVERSITY OF UTAH \\ WASHINGTON STATE UNIVERSITY \\ UNIVERSITY OF WASHINGTON
}

The Supporting Institutions listed above contribute to the cost of publication of this Journal, but they are not owners or publishers and have no responsibility for its content or policies.

Mathematical papers intended for publication in the Pacific Journal of Mathematics should be in typed form or offset-reproduced (not dittoed), double spaced with large margins. Please do not use built up fractions in the text of the manuscript. However, you may use them in the displayed equations. Underline Greek letters in red, German in green, and script in blue. The first paragraph must be capable of being used separately as a synopsis of the entire paper. In particular it should contain no bibliographic references. Please propose a heading for the odd numbered pages of less than 35 characters. Manuscripts, in triplicate, may be sent to any one of the editors. Please classify according to the 1980 Mathematics Subject Classification (1985 Revision) scheme which can be found in the December index volumes of Mathematical Reviews. Supply name and address of author to whom proofs should be sent. All other communications should be addressed to the managing editor, or Elaine Barth, University of California, Los Angeles, California 90024-1555-05.

There are page-charges associated with articles appearing in the Pacific Journal of Mathematics. These charges are expected to be paid by the author's University, Government Agency or Company. If the author or authors do not have access to such Institutional support these charges are waived. Single authors will receive 50 free reprints; joint authors will receive a total of 100 free reprints. Additional copies may be obtained at cost in multiples of 50 .

The Pacific Journal of Mathematics is issued monthly as of January 1966. Regular subscription rate: $\$ 190.00$ a year (6 Vols., 12 issues). Special rate: $\$ 95.00$ a year to individual members of supporting institutions.

Subscriptions, orders for numbers issued in the last three calendar years, and changes of address should be sent to Pacific Journal of Mathematics, P.O. Box 969, Carmel Valley, CA 93924, U.S.A. Old back numbers obtainable from Kraus Periodicals Co., Route 100, Millwood, NY 10546.

The Pacific Journal of Mathematics at P.O. Box 969, Carmel Valley, CA 93924 (ISSN 0030-8730) is published monthly. Second-class postage paid at Carmel Valley, California 93924, and additional mailing offices. Postmaster: send address changes to Pacific Journal of Mathematics, P.O. Box 969, Carmel Valley, CA 93924.

\section{PUBLISHED BY PACIFIC JOURNAL OF MATHEMATICS, A NON-PROFIT CORPORATION}




\section{Pacific Journal of Mathematics}

\section{Vol. 143, No. $2 \quad$ April, 1990}

Gustavo Corach, Horacio Porta and Lázaro Recht, Differential geometry of systems of projections in Banach algebras ................. 209

Peter Fleischmann and Jens Carsten Jantzen, Simple periodic modules of

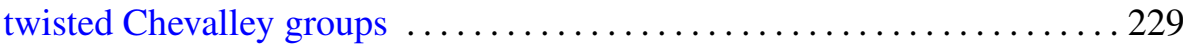

Niels Gronbaek, Amenability of discrete convolution algebras, the commutative case ...................................243

Nguyên H. V. Hung, The mod 2 equivariant cohomology algebras of

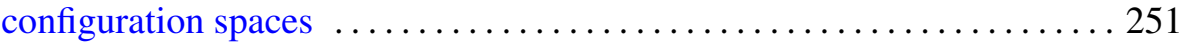

Wojciech Kucharz, Global almost analytic algebraicity of analytic sets . . . 287 John Merrill, A class of consistent anti-Martin's axioms .............. 301

Takafumi Murai, The power 3/2 appearing in the estimate of analytic

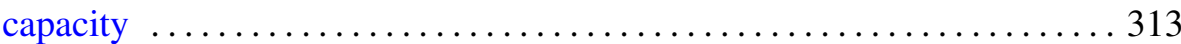

L. Panaitopol and Doru Stefanescu, On the generalized difference

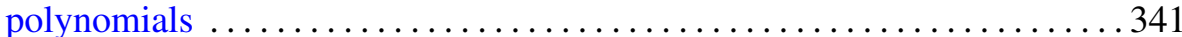

Katsuhiro Shiohama, Takashi Shioya and Minoru Tanaka, Mass of rays

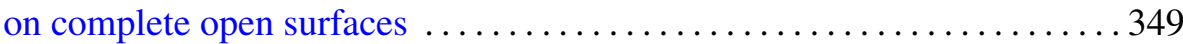

Gerhard Ströhmer, About compressible viscous fluid flow in a bounded

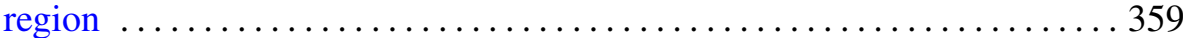

A. Ülger, Arens regularity sometimes implies the RNP 